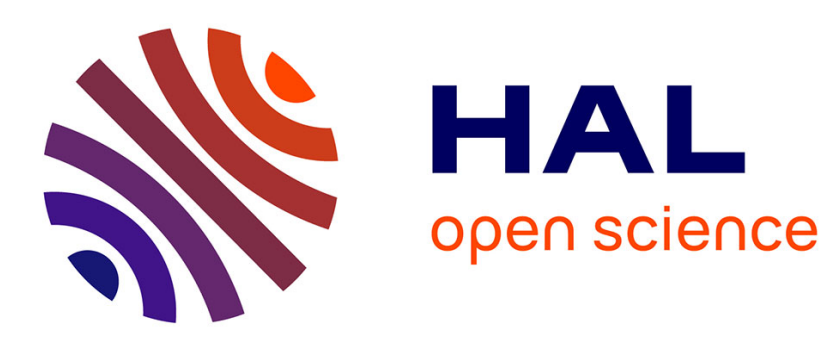

\title{
Calcul théorique des intensités de bandes du premier système négatif de l'azote
}

R. Grandmontagne, A.-M. Jorus, F. Vincent

\section{To cite this version:}

R. Grandmontagne, A.-M. Jorus, F. Vincent. Calcul théorique des intensités de bandes du premier système négatif de l'azote. Journal de Physique, 1970, 31 (8-9), pp.749-753. 10.1051/jphys:019700031089074900 . jpa-00206975

\section{HAL Id: jpa-00206975 https://hal.science/jpa-00206975}

Submitted on 1 Jan 1970

HAL is a multi-disciplinary open access archive for the deposit and dissemination of scientific research documents, whether they are published or not. The documents may come from teaching and research institutions in France or abroad, or from public or private research centers.
L'archive ouverte pluridisciplinaire HAL, est destinée au dépôt et à la diffusion de documents scientifiques de niveau recherche, publiés ou non, émanant des établissements d'enseignement et de recherche français ou étrangers, des laboratoires publics ou privés. 


\title{
CALCUL THÉORIQUE DES INTENSITÉS DE BANDES DU PREMIER SYSTĖME NÉGATIF DE L'AZOTE
}

\author{
R. GRANDMONTAGNE, A.-M. JORUS, F. VINCENT
}

Laboratoire de Spectroscopie de la Faculté des Sciences de Lyon, 43, Boulevard du 11-Novembre-1918, 69, Villeurbanne

(Reçu le 16 mars 1970)

\begin{abstract}
Résumé. - Nous présentons ici une détermination de facteurs de Franck-Condon et de $r$-centroïds, pour des transitions entre niveaux de vibration élevés $\left(v^{\prime} \leqslant 20\right.$, et $\left.v^{\prime \prime} \leqslant 17\right)$; les fonctions d'onde utilisées ont été calculées par Eido, en se servant d'une courbe de potentiel empirique, satisfaisant à la quantification de l'action de Rydberg, et à la valeur expérimentale de la constante de rotation.

Abstract. - We present a determination of Franck-Condon factors and $r$-centroids for transitions between high vibrational levels $\left(v^{\prime} \leqslant 20\right.$ and $\left.v^{\prime \prime} \leqslant 17\right)$. The wave functions used have been computed by Eido, using empirical potential curves, which satisfy the quantisation of Rydberg's action and the experimental value of the rotational constant.
\end{abstract}

Entre deux états de vibration, l'un supérieur de nombre quantique de vibration $v^{\prime}$, l'autre inférieur de nombre quantique $v^{\prime \prime}$, l'intensité de la bande émise, s'exprime par [1]:

$$
I\left(v^{\prime}, v^{\prime \prime}\right)=K\left(v^{\prime}\right) v^{4}\left(v^{\prime}, v^{\prime \prime}\right)\left|\int \Psi_{v^{\prime}} \Psi_{v^{\prime \prime}} \mathrm{d} r\right|^{2} R e^{2} .
$$

Dans cette relation $v\left(v^{\prime}, v^{\prime \prime}\right)$ est la fréquence de la bande émise, $K\left(v^{\prime}\right)$ est un terme constant spécifique de l'état supérieur de la transition et vaut $64 \pi^{4} c N\left(v^{\prime}\right) / 3$ où $N\left(v^{\prime}\right)$ représente le nombre total de molécules dans l'état supérieur $v^{\prime}$.

La quantité

$$
\left|\int \Psi_{v^{\prime}} \Psi_{v^{\prime \prime}} \mathrm{d} r\right|^{2}=q_{v^{\prime} v^{\prime \prime}}
$$

est le facteur de Franck-Condon de la transition. Dans l'expression de $I\left(v^{\prime}, v^{\prime \prime}\right)$ on ignore la dépendance de $R e$ avec la distance internucléaire $r$. Condon [2] a admis que l'on pouvait remplacer $R e$ par une valeur moyenne ; Nicholls et Jarmain [3] ont proposé de prendre la valeur $\operatorname{Re}(r)$ pour une distance internucléaire spécifique de la transition, appelée $r$-centroïd, et définie par:

$$
\bar{r}_{v^{\prime} v^{\prime \prime}}=\frac{\int \Psi_{v^{\prime}} r \Psi_{v^{\prime \prime}} \mathrm{d} r}{\int \Psi_{v^{\prime}} \Psi_{v^{\prime \prime}} \mathrm{d} r}
$$

en adoptant cette idée, le facteur $R e^{2}$ devient

$$
\operatorname{Re}^{2}\left(\bar{r}_{v^{\prime} v^{\prime \prime}}\right) \text {. }
$$

Pour la détermination théorique de l'intensité de la transition $\left(v^{\prime}, v^{\prime \prime}\right)$ la connaissance du facteur de Franck-Condon et du $r$-centroïd, quantités spécifiques de la transition, seront donc d'une importance fondamentale.

Nous décrirons dans les paragraphes suivants la méthode optico-électrique nouvelle qui nous a permis d'évaluer les facteurs de Franck-Condon et les $r$-centroïds relatifs à la transition entre les niveaux $v^{\prime}$ de vibration de l'état $B^{2} \Sigma_{u}^{+}$et les niveaux $v^{\prime \prime}$ de vibration de l'état fondamental $X^{2} \Sigma_{g}^{+}$de $N_{2}^{+}$.

Les fonctions d'onde utilisées sont les fonctions obtenues par Eido [4] par intégration directe sur ordinateur de l'équation de Schrodinger. Eido utilise pour cette intégration une courbe potentielle déterminée graphiquement à partir des constantes de rotation et des termes spectraux expérimentaux, sans hypothèse sur l'équation mathématique de cette courbe. Ainsi les fonctions d'onde utilisées peuvent être dites «réelles".

Principe de la méthode. - Pour la détermination des facteurs de Franck-Condon, il était nécessaire de posséder des fonctions d'onde normées. Or, les fonctions d'onde d'Eido ne sont pas normées; elles se présentent sous forme de graphiques d'ordonnées $y^{\prime}$ et $y^{\prime \prime}$ relatives à la même abscisse ; la détermination de $q_{v^{\prime} v^{\prime \prime}}$ se ramènera donc à l'évaluation de trois intégrales de recouvrement :

$$
\begin{gathered}
A_{N v^{\prime}}=\left|\int y^{\prime 2} \mathrm{~d} r\right| \quad A_{N v^{\prime \prime}}=\left|\int y^{\prime \prime 2} \mathrm{~d} r\right| \\
A_{v^{\prime} v^{\prime \prime}}=\left|\int y^{\prime} y^{\prime \prime} \mathrm{d} r\right|
\end{gathered}
$$


TABLEAU I

Facteurs de Franck-Condon et r-centroïds pour le premier système négatif de l'azote $B^{2} \Sigma_{u}^{+}-X^{2} \Sigma_{g}^{+}$

\begin{tabular}{|c|c|c|c|c|c|c|c|c|c|}
\hline$v^{\prime} / v^{\prime \prime}$ & 0 & 1 & 2 & 3 & 4 & 5 & 6 & 7 & 8 \\
\hline - & - & - & - & - & - & - & - & - & - \\
\hline \multirow[b]{2}{*}{0} & 0,672 & 0,291 & 0,081 & 0,014 & 0,006 & 0,000 & & & \\
\hline & 1,115 & 1,058 & 1,006 & 0,976 & 0,987 & & & & \\
\hline \multirow{2}{*}{1} & 0,307 & 0,257 & 0,289 & 0,135 & 0,041 & 0,009 & 0,002 & 0,000 & \\
\hline & 1,155 & 1,102 & 1,062 & 0,998 & 0,983 & 0,947 & 0,913 & & \\
\hline \multirow{2}{*}{2} & 0,040 & 0,398 & 0,088 & 0,250 & 0,147 & 0,071 & 0,025 & 0,004 & 0,000 \\
\hline & 1,246 & 1,161 & 1,124 & 1,053 & 1,006 & 0,984 & 0,971 & & \\
\hline \multirow{2}{*}{3} & 0,007 & 0,094 & 0,400 & 0,026 & 0,194 & 0,160 & 0,087 & 0,022 & 0,009 \\
\hline & 1,263 & 1,230 & 1,174 & 1,187 & 1,061 & 1,005 & 0,992 & 0,926 & 0,938 \\
\hline \multirow{2}{*}{4} & & 0,007 & 0,120 & 0,390 & 0,002 & 0,094 & 0,159 & 0,097 & 0,045 \\
\hline & & 1,261 & 1,247 & 1,183 & 1,537 & 1,058 & 1,036 & 1,007 & 0,940 \\
\hline \multirow[b]{2}{*}{5} & & & 0,011 & 0,159 & 0,426 & 0,001 & 0,046 & 0,131 & 0,100 \\
\hline & & & 1,342 & 1,245 & 1,206 & & 1,054 & 1,043 & 0,999 \\
\hline \multirow{2}{*}{6} & & & & 0,015 & 0,218 & 0,410 & 0,007 & 0,037 & 0,131 \\
\hline & & & & 1,316 & 1,255 & 1,209 & 0,951 & 1,125 & 1,001 \\
\hline \multirow{2}{*}{7} & & & & 0,000 & 0,007 & 0,174 & 0,391 & 0,014 & 0,059 \\
\hline & & & & 1,123 & 1,438 & 1,293 & 1,305 & 1,024 & 1,168 \\
\hline \multirow{2}{*}{8} & & & & 0,000 & 0,001 & 0,003 & 0,295 & 0,460 & 0,019 \\
\hline & & & & 1,368 & 1,268 & & 1,400 & 1,313 & \\
\hline \multirow{2}{*}{9} & & & & & & 0,003 & 0,003 & 0,215 & 0,498 \\
\hline & & & & & & & 0,846 & 1,399 & 1,316 \\
\hline 10 & & & & & & & 0,007 & 0,027 & 0,104 \\
\hline \multirow[b]{2}{*}{11} & & & & & & & & 0014 & 1,420 \\
\hline & & & & & & & 0,002 & 1,387 & 1,243 \\
\hline \multirow{2}{*}{12} & & & & & & & & 0,002 & 0,001 \\
\hline & & & & & & & & 1,130 & \\
\hline 13 & & & & & & & & 0,001 & 0,008 \\
\hline 14 & & & & & & & & & $\begin{array}{l}1,296 \\
0,003\end{array}$ \\
\hline
\end{tabular}

Sur la première ligne on trouve les facteurs de Franck-Condon.

Sur la deuxième ligne on trouve le $r$-centroïd exprimé en Angstrom.

d'où

$$
q_{v^{\prime} v^{\prime \prime}}=\frac{A_{v^{\prime} v^{\prime \prime}}^{2}}{A_{N v^{\prime}} A_{N v^{\prime \prime}}}
$$

Par contre, la détermination du $r$-centroïd ne nécessite pas de fonctions d'onde normées, mais seulement la connaissance des intégrales :

$$
\int y^{\prime} y^{\prime \prime} \mathrm{d} r \quad \text { et } \quad \int y^{\prime} r y^{\prime \prime} \mathrm{d} r \text {. }
$$

On aura ainsi :

$$
\bar{r}_{v^{\prime} v^{\prime \prime}}=\frac{\int y^{\prime} r y^{\prime \prime} \mathrm{d} r}{\int y^{\prime} y^{\prime \prime} \mathrm{d} r} .
$$

Il suffit par conséquent d'étudier ces intégrales dans des conditions identiques.

L'appareillage expérimental [5] se compose de trois parties :

- un multiplicateur optique,

- un multiplicateur électrique,

- un intégrateur électrique.

1. Principe De LA MUltiplication optiQue (Facteurs de Franck-Condon). - Les courbes d'ordonnées $y^{\prime}$ et $y^{\prime \prime}$ sont reproduites sur film transparent, la partie comprise entre l'axe des $r$ et la courbe reste transparente à la lumière, la partie extérieure à la courbe est opaque à la lumière.

Ces films sont placés sur un chariot, qui se déplace à vitesse uniforme, dans le champ d'un faisceau lumineux, les abscisses sur les deux films sont à chaque 
TABLEAU I (suite)

Facteurs de Franck-Condon et r-centroïds

\begin{tabular}{|c|c|c|c|c|c|c|c|c|c|}
\hline$v^{\prime} / v^{\prime \prime}$ & 9 & 10 & 11 & 12 & 13 & 14 & 15 & 16 & 17 \\
\hline- & - & - & - & - & - & - & - & - & - \\
\hline \multicolumn{10}{|l|}{0} \\
\hline \multicolumn{10}{|l|}{1} \\
\hline \multicolumn{10}{|l|}{2} \\
\hline \multirow{2}{*}{3} & 0,006 & 0,003 & & & & & & & \\
\hline & 0,979 & 0,995 & & & & & & & \\
\hline \multirow{2}{*}{4} & 0,015 & 0,008 & 0,003 & & & & & & \\
\hline & 0,916 & 0,934 & & & & & & & \\
\hline \multirow{2}{*}{5} & 0,046 & 0,028 & 0,009 & & & & & & \\
\hline & 0,951 & 0,968 & 0,974 & & & & & & \\
\hline \multirow{2}{*}{6} & 0,100 & 0,060 & 0,026 & 0,000 & & & & & \\
\hline & 1,044 & 1,013 & 0,988 & & & & & & \\
\hline \multirow{2}{*}{7} & 0,100 & 0,105 & 0,067 & 0,000 & & & & & \\
\hline & 1,093 & 1,096 & 1,049 & & & & & & \\
\hline \multirow{2}{*}{8} & 0,042 & 0,112 & 0,083 & 0,089 & & & & & \\
\hline & 1,031 & 1,114 & 1,079 & 0,973 & & & & & \\
\hline \multirow{2}{*}{9} & 0,009 & 0,020 & 0,119 & 0,097 & 0,088 & & & & \\
\hline & 1,668 & 0,901 & 1,132 & 0,986 & 0,965 & & & & \\
\hline \multirow{2}{*}{10} & 0,598 & 0,139 & 0,023 & 0,075 & 0,041 & 0,061 & 0,021 & 0,010 & 0,005 \\
\hline & 1,276 & 1,499 & 1,679 & & & 1,065 & & & \\
\hline \multirow{2}{*}{11} & 0,064 & 0,568 & 0,249 & 0,045 & 0,099 & 0,014 & 0,057 & 0,009 & 0,010 \\
\hline & 1,580 & 1,306 & 1,466 & & & & 1,121 & & \\
\hline \multirow{2}{*}{12} & 0,079 & 0,029 & 0,469 & 0,192 & 0,027 & 0,225 & 0,009 & 0,076 & 0,009 \\
\hline & 1,286 & 1,084 & 1,291 & 1,422 & 1,744 & 1,232 & & 1,098 & 0,589 \\
\hline \multirow{2}{*}{13} & 0,011 & 0,084 & 0,116 & 0,238 & 0,168 & 0,056 & 0,310 & 0,001 & 0,051 \\
\hline & 1,127 & 1,323 & 1,222 & 1,269 & & & 1,275 & & \\
\hline \multirow{2}{*}{14} & 0,004 & 0,041 & 0,065 & 0,344 & 0,012 & 0,143 & 0,034 & 0,345 & 0,003 \\
\hline & & 1,191 & 1,416 & 1,258 & & & & 1,311 & \\
\hline \multirow{2}{*}{15} & & 0,001 & 0,070 & 0,007 & 0,360 & 0,028 & 0,035 & 0,021 & 0,259 \\
\hline & & & 1,247 & 1,599 & 1,278 & & & & 1,307 \\
\hline \multirow{2}{*}{16} & & 0,002 & 0,008 & 0,035 & 0,110 & 0,080 & 0,086 & 0,002 & 0,010 \\
\hline & & & 1,106 & 1,314 & 1.218 & 1,264 & & & 0,964 \\
\hline \multirow{2}{*}{17} & & 0,003 & 0,001 & 0,038 & 0,006 & 0,130 & 0,008 & 0,066 & 0,007 \\
\hline & & & & 1,196 & 0,980 & 1,240 & & & \\
\hline \multirow{2}{*}{18} & & 0,001 & 0,004 & 0,010 & 0,040 & 0,028 & 0,110 & 0,020 & 0,016 \\
\hline & & & 1,284 & 1,214 & 1,303 & 1,199 & 1,250 & & \\
\hline \multirow{2}{*}{19} & & & & 0,000 & 0,039 & 0,014 & 0,098 & 0,040 & 0,048 \\
\hline & & & & & 1,229 & 1,431 & 1,297 & 1,248 & 1,324 \\
\hline \multirow{2}{*}{20} & & & & 0,004 & 0,008 & 0,034 & 0,010 & 0,073 & 0,010 \\
\hline & & & & & 1,222 & 1,318 & 1,182 & 1,260 & 1,378 \\
\hline
\end{tabular}

instant les mêmes. Le faisceau lumineux est diaphragmé par l'un des films puis par l'autre, de telle sorte qu'un photomultiplicateur donne un courant de sortie proportionnel au produit $y^{\prime} y^{\prime \prime}$.

Un système d'inverseur permet de tenir compte du signe positif ou négatif des ordonnées des courbes $y^{\prime}$ et $y^{\prime \prime}$.

Le courant photomultiplié est alors envoyé dans un intégrateur, qui délivre une tension finale proportionnelle à l'intégrale $\int y^{\prime} y^{\prime \prime} \mathrm{d} r$.

Pour l'évaluation des facteurs de Franck-Condon l'appareillage se réduit au dispositif de multiplication optique et à l'intégrateur.

2. Principe de multiplication électrique [6] ( $r$-centroïds). - Le courant du photomultiplicateur passe dans un changeur de signe, qui agit comme adaptateur d'impédance, et permet d'avoir à sa sortie une tension indépendante de sa charge.

Un potentiomètre variable, solidaire de l'axe du moteur qui entraîne le chariot portant les films des fonctions d'onde, est alors soumis à la tension précédente. Une résistance constante en série avec celle 
du potentiomètre permet d'obtenir une résistance variable proportionnelle à chaque instant à la distance internucléaire. On obtient ainsi à la sortie du potentiomètre une quantité proportionnelle à $y^{\prime} r y^{\prime \prime}$.

L'intégrateur électronique fournit une tension de sortie proportionnelle à

$$
\int y^{\prime} y^{\prime \prime} \mathrm{d} r \quad \text { ou à } \quad \int y^{\prime} r y^{\prime \prime} \mathrm{d} r .
$$

Par cette méthode nous avons déterminé les factures de Franck-Condon et les $\bar{r}$-centroïds de la transitions $B^{2} \Sigma_{u}^{+}-X^{2} \Sigma_{g}^{+}$de $N_{2}^{+}$, pour des niveaux $v^{\prime}$ de 0 à 20 et $v^{\prime \prime}$ de 0 à 17 .

Le tableau I donne pour chaque transition $\left(v^{\prime}, v^{\prime \prime}\right)$ le facteur de Franck-Condon et le $\bar{r}$-centroid évalués par la méthode optico-électrique. La comparaison de ces résultats avec ceux de Nicholls [7] et ceux de Wallace et Nicholls [8] montre une bonne coïncidence.

La distribution des maxima des facteurs de FranckCondon met en évidence une parabole principale de Condon ayant son axe parallèle à la première diagonale. Une parabole secondaire pratiquement confondue avec la première diagonale du tableau, apparaît pour des niveaux de vibration supérieure à $v=13$.

Les facteurs de Franck-Condon représentant des intégrales de recouvrement de fonctions d'onde normées, la somme de ces facteurs par progression doit être égale à 1 . Ce test, dit test de Bates, appliqué ici, permet d'évaluer une incertitude inférieure à $5 \%$.

Les tableaux II et III comportent d'une part les intensités théoriques déduites du facteur de FranckCondon, par multiplication par $K\left(v^{\prime}\right) v^{4}, K$ étant proportionnel à la population du niveau initial ; d'autre part les intensités expérimentales données par Herzberg [9], par Janin et Eyraud [10] et d'autres auteurs [11]. La comparaison des intensités théoriques aux résultats expérimentaux, a été faite par progression $v^{\prime}=$ constante, sans tenir compte du facteur $K$, représentatif de la population de l'état supérieur; à chaque fois un changement d'échelle des intensités expérimentales a été réalisé pour permettre la comparaison.

Le tableau montre que dans la totalité de l'étude une concordance visible est constatée toutes les fois que les intensités expérimentales sont données. Cependant, dès le niveau $v^{\prime}=4$ certaines bandes, qui d'après notre étude auraient des intensités relativement élevées n'ont pas été mises en évidence expérimentalement.

A partir du niveau $v^{\prime}=10$, les intensités observées visuellement étant données par plusieurs auteurs, il est assez difficile de faire une comparaison soignée; mais malgré tout, dans les zones observées, certaines anomalies existent ; ainsi dans la progression $v^{\prime}=16$ la bande $(16,11)$ semble avoir une intensité moins

TABLEAU II

Comparaison des intensités

\begin{tabular}{|c|c|c|c|c|c|c|c|c|c|c|c|c|c|}
\hline$v^{\prime} / v^{\prime \prime}$ & 0 & 1 & 2 & 3 & 4 & 5 & 6 & 7 & 8 & 9 & 10 & 11 & 12 \\
\hline 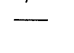 & - & - & - & - & - & - & - & - & - & - & - & 一 & - \\
\hline \multirow{2}{*}{0} & 286 & 87 & 16 & 2 & 0,5 & & & & & & & & \\
\hline & 286 & 76 & 9,5 & 0,3 & & & & & & & & & \\
\hline \multirow{2}{*}{1} & 186 & 113 & 91 & 29 & 6 & 1 & & & & & & & \\
\hline & 186 & 155 & 155 & 31 & 2,5 & 0,3 & & & & & & & \\
\hline \multirow{2}{*}{2} & 33 & 246 & 40 & 80 & 33 & 11 & 2 & 0 & & & & & \\
\hline & & 246 & 32,8 & 82 & 41 & 4,1 & 0,6 & & & & & & \\
\hline \multirow{2}{*}{3} & 8 & 79 & 252 & 12 & 64 & 37 & 14 & 3 & 0,6 & 0 & & & \\
\hline & 1,3 & 75,6 & 252 & & 38,2 & 38 & 4 & 0,6 & & & & & \\
\hline \multirow{2}{*}{4} & & 8 & 102 & 248 & 1 & 32 & 38 & 16 & 5 & 1 & 0 & & \\
\hline & & 2,5 & 198 & 248 & & 50 & 87 & 12,4 & 2,48 & & & & \\
\hline \multirow{2}{*}{5} & & & & 135 & 273 & 0,5 & 16 & 32 & 17 & 5 & 2 & 0 & \\
\hline & & & & & 273 & & 17,5 & 30 & 10,9 & 2 & & & \\
\hline \multirow[t]{2}{*}{6} & & & & 16 & 185 & 263 & 3 & 13 & 33 & 17 & 8 & 2 & \\
\hline & & & & & & & & 3,3 & 33 & 6,6 & 1 & & \\
\hline \multirow[t]{2}{*}{7} & & & & & 7 & 145 & 248 & 7 & 21 & 25 & 19 & 8 & \\
\hline & & & & & 1 & 3 & 241 & 286 & 9 & $\begin{array}{l}25 \\
14\end{array}$ & $\begin{array}{l}15 \\
28\end{array}$ & $\begin{array}{c}3,5 \\
15\end{array}$ & 11 \\
\hline 8 & & & & & & & & & & & & 15 & \\
\hline 9 & & & & & 15 & 4 & 3 & 185 & 300 & 4 & 7 & 29 & 17 \\
\hline
\end{tabular}

Sur la première ligne figurent les intensités théoriques et sur la deuxième ligne les intensités déterminées expérimentalement par Herzberg [9].

Les valeurs en italique correspondent à une remise à l'échelle des valeurs expérimentales pour faciliter la comparaison. 
TABLEAU III

Comparaison des intensités

\begin{tabular}{|c|c|c|c|c|c|c|c|c|c|c|c|c|c|}
\hline$v^{\prime} / v^{\prime \prime}$ & 5 & 6 & 7 & 8 & 9 & 10 & 11 & 12 & 13 & 14 & 15 & 16 & 17 \\
\hline- & - & - & - & - & - & - & - & - & - & - & - & - & - \\
\hline 10 & 11 & 9 & 26 & 79 & 351 & 61 & 7 & 18 & 7 & 7 & 2 & 0 & 0 \\
\hline 11 & 4 & 3 & 16 & 21 & $\begin{array}{l}46 \\
46\end{array}$ & $\begin{array}{r}61 \\
313\end{array}$ & 104 & 14 & 23 & $\begin{array}{l}7 \\
2\end{array}$ & 6 & 1 & 0 \\
\hline \multirow{2}{*}{12} & & & 3 & 1 & 7 & 19 & 241 & 59 & 8 & 48 & 1 & 8 & \\
\hline & & & 3 & & & $40^{*}$ & $241^{*}$ & & & & & & \\
\hline \multirow{2}{*}{13} & & & 2 & 10 & 11 & 67 & 72 & 113 & 60 & 15 & 61 & 0 & 5 \\
\hline & & & & 0 & 0 & 67 & & $113^{*}$ & & & 42 & & \\
\hline \multirow{2}{*}{14} & & & & 4 & 5 & 38 & 47 & 195 & 9 & 47 & 8 & 62 & 0 \\
\hline & & & & & & 38 & $49 *$ & $195^{*}$ & & & & $390 *$ & \\
\hline \multirow{2}{*}{15} & & & & & & 1 & 59 & 5 & 186 & 11 & 10 & 5 & 42 \\
\hline & & & & & & & & 0 & $186^{*}$ & & & & 42 \\
\hline \multirow{2}{*}{16} & & & & & & 2 & 8 & 27 & 66 & 37 & 31 & 0 & 2 \\
\hline & & & & & & & 28 & 18,5 & $66^{*}$ & 37 & & & 9,25 \\
\hline \multirow[t]{2}{*}{17} & & & & & & 4 & 1 & 33 & 4 & 71 & 3 & 21 & 2 \\
\hline & & & & & & & & 33 & & 29 & & & \\
\hline \multirow[t]{2}{*}{18} & & & & & & 1 & 5 & 10 & 32 & 17 & 54 & 7 & 4 \\
\hline & & & & & & & 10 & 0 & 40 & 0 & 40 & & \\
\hline 19 & & & & & & & 3 & & 24 & $\begin{array}{l}10 \\
12\end{array}$ & 21 & 17 & 10 \\
\hline \multirow{2}{*}{20} & & & & & & & 1 & 5 & 8 & 27 & 6 & 36 & 4 \\
\hline & & & & & & & & & 14 & 28 & & 36 & 14 \\
\hline
\end{tabular}

Sur la première ligne figurent les intensités théoriques et sur la deuxième ligne les intensités estimées visuellement par J. Janin et I. Eyraud [10].

Les bandes marquées d'un astérisque ont été obtenues par Parker, par Smyth et Arnott ou par Douglas [11].

grande que la bande $(16,12)$; or expérimentalement l'inverse a été observé. Il en est de même pour les bandes $(17,12)$ et $(17,14)$ et pour les bandes $(19,13)$ et $(19,15)$.

Le travail actuel recouvre pratiquement tout le domaine spectral étudié expérimentalement par Janin et Eyraud.

Il exploite essentiellement des fonctions d'onde ne dépendant d'aucune contrainte mathématique, imposée $a$ priori à la courbe de potentiel.

\section{Bibliographie}

[1] Herzberg (G.), Spectra of diatomic molecules, 1955.

[2] Condon (E. U), Phys. Rev., 1928, 32, 858.

[3] Nicholls (R. W.) et Jarmain (W. R.), Proc. Phys. Soc., 1956, 69, 253.

[4] EIDo (R.), Cahier de Physique, 1964, 165, 209. EIDo (R.), Communication privée.

[5] Jorus (A.-M.), Thèse de Doctorat de Spécialité (juin 1967).

[6] Vincent (F.), Thèse de Doctorat de Spécialité (janvier 1969).
[7] Nicholls (R. W.), J. Res. Nat. Bur. Stand., 1961, $65 \mathrm{~A}, 451$.

[8] Wallace (L. V.) et Nicholls (R. W.), J. Terr. Atm. Phys., 1955, 7, 101.

[9] Herzberg (G.), Annalen der Physik, 1928, 86, 202. [10] Janin (J.) et Eyraud (I.), J. Phys. et Rad., 1954, 15, 88.

[11] Parker (A. E.), Phys. Rev., 1933, 44, 90 et 914. SMYth (H. D.) et Arnott (E. G. F.), Phys. Rev., 1930, 36, 1023.

Douglas (A. E.), Can. J. Phys., 1952, 30, 302. 